\title{
Synthesis and mesomorphic properties of calamitic malonates and cyanoacetates tethered to 4-cyanobiphenyls
}

\author{
Katharina C. Kress, Martin Kaller, Kirill V. Axenov, Stefan Tussetschläger
} and Sabine Laschat*

\section{Full Research Paper}

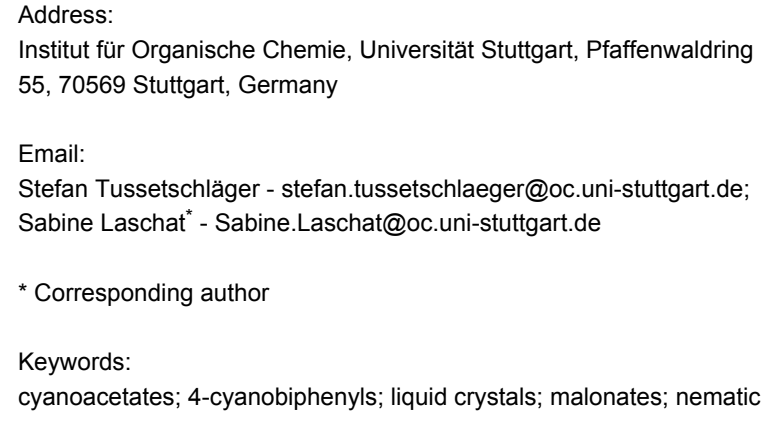

Institut für Organische Chemie, Universität Stuttgart, Pfaffenwaldring 55, 70569 Stuttgart, Germany

\section{Email:}

Stefan Tussetschläger - stefan.tussetschlaeger@oc.uni-stuttgart.de; Sabine Laschat ${ }^{*}$ - Sabine.Laschat@oc.uni-stuttgart.de

* Corresponding author

Keywords:

cyanoacetates; 4-cyanobiphenyls; liquid crystals; malonates; nematic

\author{
Beilstein J. Org. Chem. 2012, 8, 371-378. \\ doi:10.3762/bjoc. 8.40 \\ Received: 08 November 2011 \\ Accepted: 20 February 2012 \\ Published: 09 March 2012 \\ This article is part of the Thematic Series "Progress in liquid crystal \\ chemistry II". \\ Associate Editor: P. J. Skabara \\ (C) 2012 Kress et al; licensee Beilstein-Institut. \\ License and terms: see end of document.
}

\begin{abstract}
4-Cyano-1,1'-biphenyl derivatives bearing $\omega$-hydroxyalkyl substituents were reacted with methyl 3-chloro-3-oxopropionate or cyanoacetic acid, giving liquid-crystalline linear malonates and cyanoacetates. These compounds formed monotropic nematic phases at $62{ }^{\circ} \mathrm{C}$ down to ambient temperature upon cooling from the isotropic liquid. The mesomorphic properties were investigated by differential scanning calorimetry, polarizing optical microscopy and X-ray diffraction (WAXS).
\end{abstract}

\section{Introduction}

Nematic liquid crystals display mesophases in which the molecules are oriented along one vector defined by the director axis, but with the molecular arrangement in random positional order [1]. Nematic phases typically display low viscosity [2-4]. Due to the long-range orientational order they reveal anisotropic properties. These features make nematic liquid crystals very attractive materials for electronics [5-8], for the construction of liquid crystal displays [9-11], or as anisotropic conductors $[12,13]$. Over the past few decades, a huge variety of organic calamitic compounds, which form nematic liquid crystals, have been synthesized and investigated $[1,14]$.
Bulkin et al. were the first to investigate the phase behaviour of metal $\beta$-diketonate complexes such as $\mathbf{1}$ [15] (Scheme 1). Although they were not able to detect any mesophases, their study motivated others to examine the mesomorphic properties of $\beta$-diketonates in more detail [16]. Among the first examples of a nematic $\beta$-diketonate is the Cu complex 2 described by Haase [17-21]. In contrast to the various diketonato metallomesogens only a little information is available about the mesomorphic properties of metal-free diketones. Among the few examples are the nematic compounds 3 [22] and 4 [23,24] (Scheme 1). 


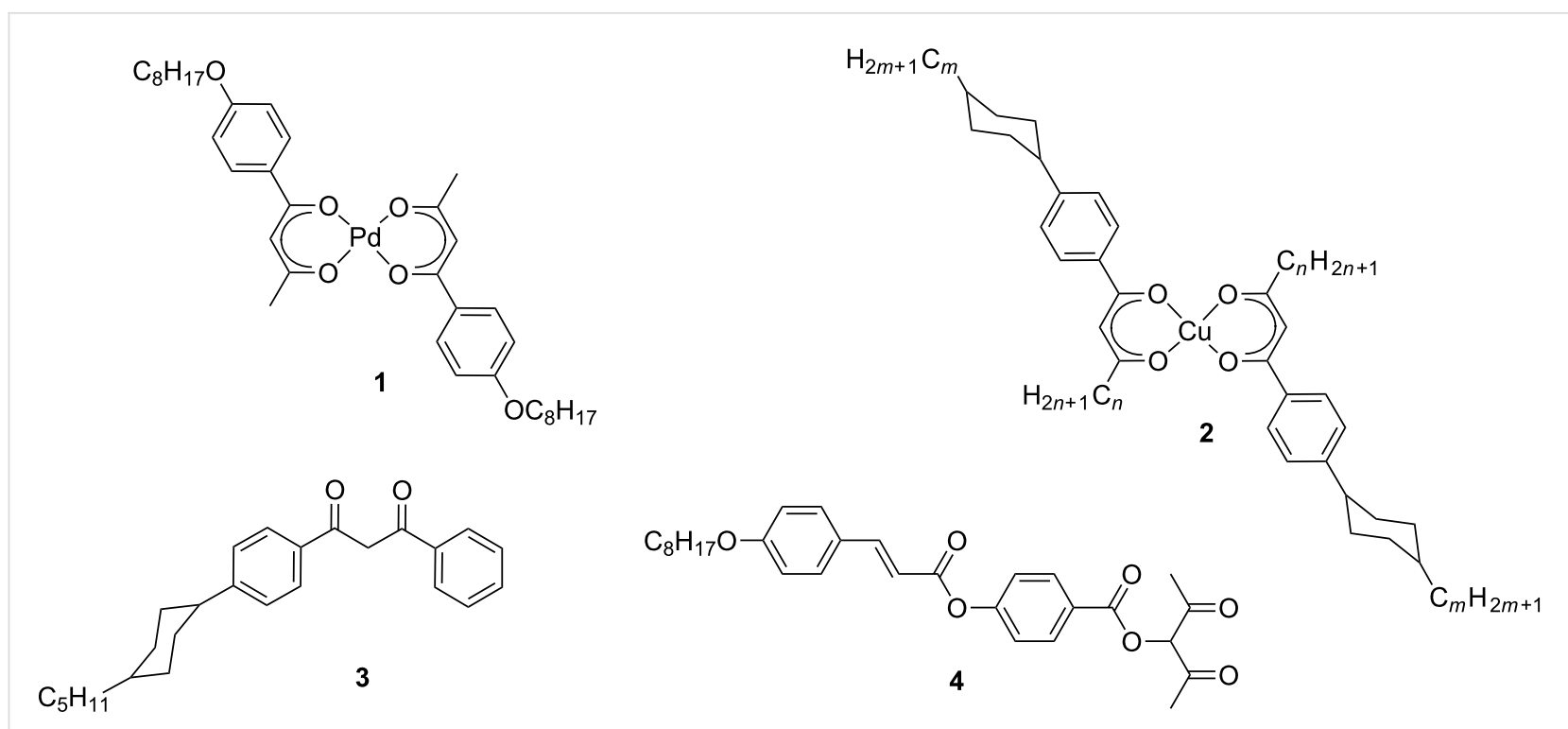

Scheme 1: Diketonato metallomesogens and diketones with mesomorphic properties.

The corresponding malonates and cyanoacetates are well known as suitable ligands for strong coordination of main-group and transition metals [25]. Benzylidene derivatives of malonic esters, so called swallow-tailed liquid crystals, were described as forming smectic phases [26]. However, most work on liquidcrystalline malonates has been devoted to $\mathrm{C}_{60}$ fullerene dendrimers [27-31]. Only a few liquid crystalline cyanoacetates have been described so far. The first example, a dihydrazide, was reported by Schubert [32]. Furthermore some calamitic and bent-core mesogens derived from $\alpha$-cyanocinnamic acid were described in the literature $[33,34]$. Therefore, we decided to explore the synthesis and mesomorphic properties of malonates and cyanoacetates 5 tethered to calamitic 4-cyanobiphenyl units (Scheme 2).

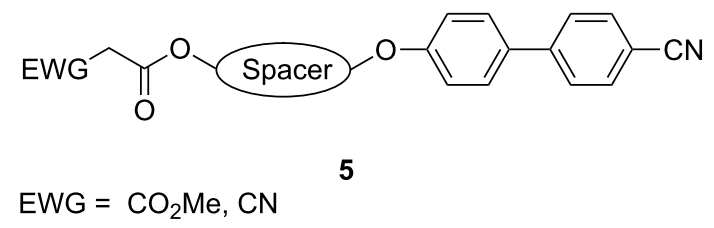

Scheme 2: Malonates and cyanoacetates tethered to calamitic 4-cyanobiphenyl units.

\section{Results and Discussion}

The syntheses of malonate and cyanoacetate derivatives 11, 13 are shown in Scheme 3. Starting from the corresponding diols 6a,b, 6-bromohexan-1-ol (7a) and 10-bromodecan-1-ol (7b) were obtained in moderate yields by bromination with aqueous $\mathrm{HBr}$ in toluene [35]. The bromides $\mathbf{7 a}, \mathbf{b}$ were reacted with 4-cyano-1,1'-biphenol (8) in acetone in the presence of $\mathrm{K}_{2} \mathrm{CO}_{3}$ giving compounds $9 \mathbf{a}, \mathbf{b}$, bearing $\mathrm{C}_{6}$ - or $\mathrm{C}_{10}$-spacers, in $68 \%$ and $60 \%$ yield, respectively [36-38] after recrystallization from methanol (Scheme 3).

The malonate unit was attached by treatment of the compounds 9a,b with methyl 3-chloro-3-oxopropionate (10) in the presence of pyridine in $\mathrm{CH}_{2} \mathrm{Cl}_{2}$ to yield the malonates $\mathbf{1 1 a}, \mathbf{b}$ in $57 \%$ and $65 \%$, respectively, after column chromatography. In a parallel approach, the precursors $9 \mathbf{a}, \mathbf{b}$ were converted to the corresponding cyanoacetates $\mathbf{1 3 a}, \mathbf{b}$ by esterification of cyanoacetic acid (12) in the presence of DMAP and dicyclohexylcarbodiimide in $\mathrm{CH}_{2} \mathrm{Cl}_{2}$. After chromatography the cyanoacetates 13a,b were isolated as colourless solids in $59 \%$ and $41 \%$ yield.

The obtained malonate and cyanoacetic esters 11a,b and 13a,b were subjected to differential scanning calorimetry (DSC) studies (Figure 1, Figure 2, and Table 1).

During the first heating runs all compounds did not show the appearance of any liquid-crystalline phase, but melted without decomposition into isotropic liquids. It was observed that the melting points increased with an increase of the spacer length between 4-cyanobiphenyl and ester groups. Thus, melting points were recorded at $49.1{ }^{\circ} \mathrm{C} / 63.4{ }^{\circ} \mathrm{C}$ for the series of $11 \mathrm{a} /$ $\mathbf{1 1 b}$ and at $89.0{ }^{\circ} \mathrm{C} / 93.2^{\circ} \mathrm{C}$ for the series of $\mathbf{1 3 a} / \mathbf{1 3 b}$, respectively (Table 1). The cyano group is a stronger electron-acceptor than the ester function, and thus the cyanoacetic ester molecules are more polarized than the corresponding malonates. Stronger dipole-dipole interactions for cyano esters $\mathbf{1 3}$ led to an increase of their clearing points compared with malonates $\mathbf{1 1 .}$ 

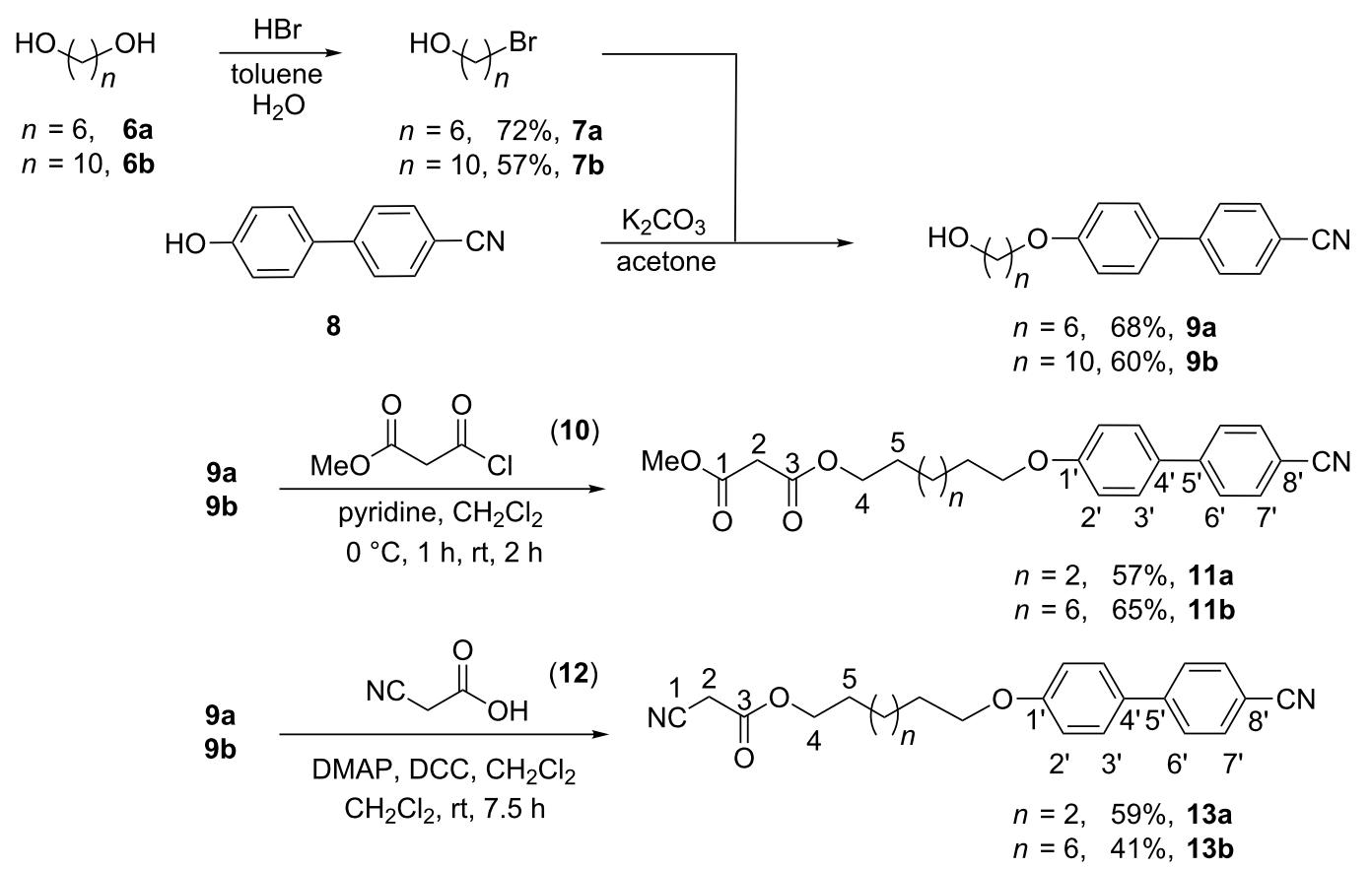

Scheme 3: Synthesis of malonate and cyanoacetates tethered to 4-cyano-biphenyl moieties.

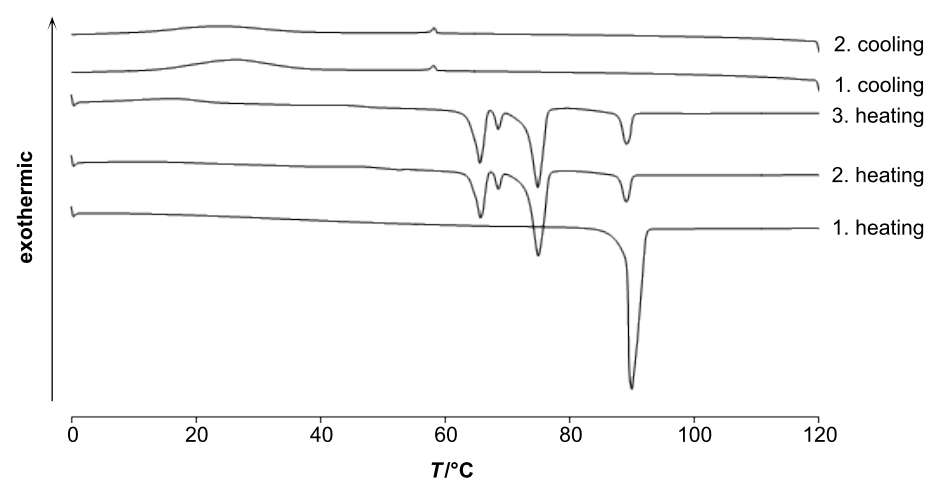

Figure 1: DSC traces of 13a (heating/cooling rate $5 \mathrm{~K} / \mathrm{min}$ ).

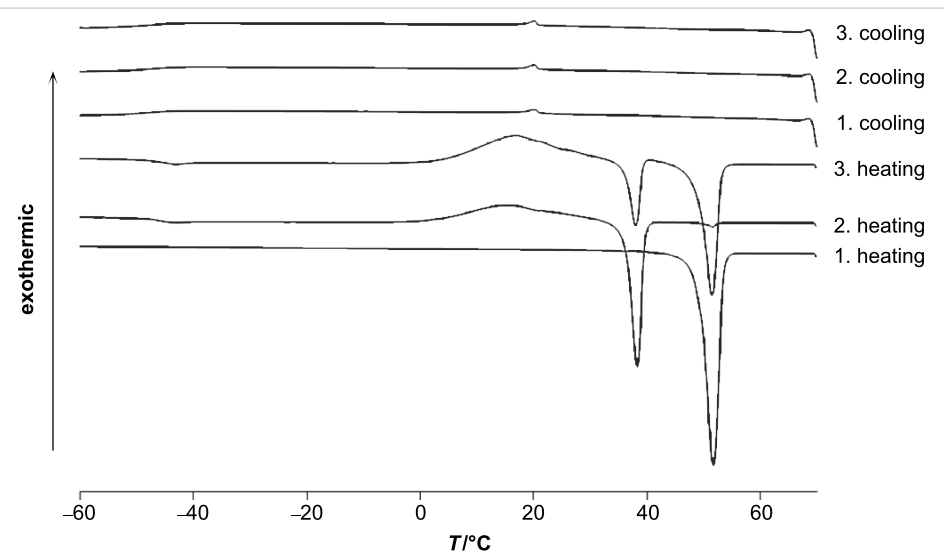

Figure 2: DSC traces of 11a (heating/cooling rate $10 \mathrm{~K} / \mathrm{min}$ ). 


\begin{tabular}{|c|c|c|c|c|c|c|c|c|c|c|c|c|}
\hline & $n$ & $\mathrm{Cr}_{1}$ & $T$ & $\Delta H$ & $\mathrm{Cr}_{2}$ & $T$ & $\Delta H$ & $\mathrm{Cr}_{3} / \mathrm{N}$ & $T$ & $\Delta H$ & I & \\
\hline \multirow[t]{5}{*}{$11 a$} & 6 & - & 49.1 & 32.4 & - & - & - & - & - & - & $\bullet$ & 1. heating ${ }^{b}$ \\
\hline & & • & - & - & - & - & - & $\mathrm{N}$ & 21.2 & -0.32 & $\bullet$ & 1. cooling $b$ \\
\hline & & • & 4.1 & -12.0 & $\bullet$ & 36.2 & 17.6 & $\mathrm{Cr}_{3}$ & 49.9 & 0.27 & • & 2. heating ${ }^{b}$ \\
\hline & & $\bullet$ & - & - & - & - & - & $N$ & 20.9 & -0.29 & • & 2. cooling ${ }^{b}$ \\
\hline & & $\bullet$ & 5.0 & -18.2 & $\bullet$ & 35.9 & 7.72 & $\mathrm{Cr}_{3}$ & 48.8 & 20.7 & • & 3. heating ${ }^{b}$ \\
\hline \multirow[t]{4}{*}{$11 \mathrm{~b}$} & 10 & • & 63.4 & 53.2 & - & - & - & - & - & - & • & 1. heating ${ }^{c}$ \\
\hline & & • & 10.0 & -24.2 & • & 14.8 & -3.45 & $\mathrm{~N}$ & 35.6 & -0.68 & • & 1. cooling ${ }^{c, d}$ \\
\hline & & $\bullet$ & 54.8 & 39.8 & - & - & - & $\mathrm{Cr}_{3}$ & 59.9 & 7.35 & • & 2. heating ${ }^{c}$ \\
\hline & & • & 14.8 & -24.2 & $\bullet$ & 31.1 & -0.33 & $N$ & 35.4 & -0.46 & • & 2. cooling ${ }^{c}$ \\
\hline \multirow[t]{5}{*}{$13 a$} & 6 & $\bullet$ & 89.0 & 39.6 & - & - & - & - & - & - & • & 1. heating ${ }^{c}$ \\
\hline & & • & - & - & - & - & - & $\mathrm{N}$ & 58.7 & -0.38 & • & 1. cooling ${ }^{C}$ \\
\hline & & • & 64.1 & 9.19 & $\bullet$ & 73.0 & 22.7 & $\mathrm{Cr}_{3}$ & 87.8 & -4.34 & • & 2. heating ${ }^{\mathrm{c}, \mathrm{e}}$ \\
\hline & & $\bullet$ & - & - & - & - & - & $N$ & 58.7 & -0.41 & • & 2. cooling ${ }^{\mathrm{C}}$ \\
\hline & & $\bullet$ & 63.9 & 11.6 & $\bullet$ & 72.9 & 19.8 & $\mathrm{Cr}_{3}$ & 87.8 & 5.48 & • & 3. heating ${ }^{\mathrm{c}, \mathrm{f}}$ \\
\hline \multirow[t]{4}{*}{$13 b$} & 10 & • & 93.2 & 49.1 & - & - & - & - & - & - & • & 1. heating ${ }^{c}$ \\
\hline & & • & 52.7 & -41.1 & - & - & - & $N$ & 61.8 & -0.71 & • & 1. cooling ${ }^{C}$ \\
\hline & & $\bullet$ & 74.7 & 13.5 & $\bullet$ & 86.3 & -7.41 & $\mathrm{Cr}_{3}$ & 91.5 & 41.5 & • & 2. heating ${ }^{c, g}$ \\
\hline & & $\bullet$ & 52.7 & -41.1 & - & - & - & $\mathrm{N}$ & 61.9 & -0.98 & $\bullet$ & 2. cooling ${ }^{\mathrm{C}}$ \\
\hline
\end{tabular}

${ }^{a} \mathrm{Cr}$ crystalline; N nematic; I isotropic; $\bullet$ phase was observed; - phase was not observed. bHeating and cooling rate: $10 \mathrm{~K} / \mathrm{min}$. ${ }^{\mathrm{CH}} \mathrm{Heating}$ and cooling rate: $5 \mathrm{~K} / \mathrm{min}$. ${ }^{\mathrm{d}}$ Another crystal-to-crystal transition $\left(31.2^{\circ} \mathrm{C},-0.24 \mathrm{~kJ} / \mathrm{mol}\right)$ was observed. ${ }^{\mathrm{e}}$ Another crystal-to-crystal transition $\left(67.7^{\circ} \mathrm{C}, 1.62 \mathrm{~kJ} / \mathrm{mol}\right)$ was observed. ${ }^{\mathrm{f}}$ Another crystal-to-crystal transition $\left(67.6^{\circ} \mathrm{C}, 1.86 \mathrm{~kJ} / \mathrm{mol}\right)$ was observed. ${ }^{\mathrm{g}}$ Another crystal-to-crystal transition $\left(78.1^{\circ} \mathrm{C}, 21.0 \mathrm{~kJ} / \mathrm{mol}\right)$ was observed.

The additional $\mathrm{Cr} \rightarrow \mathrm{Cr}$ transitions in the 2 nd and 3rd heating curves (Figure 1 and Figure 2) are probably due to equilibration and the presence of keto-enol tautomers. Molecular geometry phase-behaviour relationships in keto-enamine/imino-enol tautomers of ferrocenophanes have been previously studied by Galyametdinov [39]. In the first cooling runs the appearance of nematic mesophases was observed for both series $11 \mathbf{a}, \mathbf{b}$ and 13a,b. All compounds displayed small transition enthalpies in a range between -0.3 and $-0.7 \mathrm{~kJ} / \mathrm{mol}$ (Table 1 ) for the transition from the isotropic liquid to the corresponding mesophases. While $\mathrm{C}_{6}$-linked compounds displayed monotropic nematic mesophases at temperatures of $21^{\circ} \mathrm{C}$ for $11 \mathrm{a}$ and $59^{\circ} \mathrm{C}$ for $\mathbf{1 3 a}$, their longer and more flexible $\mathrm{C}_{10}$-linked homologues showed higher transition temperatures at $36{ }^{\circ} \mathrm{C}$ for $11 \mathrm{~b}$ and $62{ }^{\circ} \mathrm{C}$ for 13b upon cooling from the isotropic liquid. Due to supercooling, no crystallisation could be observed for compound 11a and, therefore, no mesophase range could be determined. But the second and third heating runs of 11a showed broad recrystallization peaks (Figure 2).

\section{Polarizing optical microscope (POM) studies}

POM observations of compounds $11 \mathbf{a}, \mathbf{b}$ and $\mathbf{1 3 a}, \mathbf{b}$ revealed textures typical for nematic phases, only upon cooling from the isotropic liquid (Figure 3 and Figure 4).
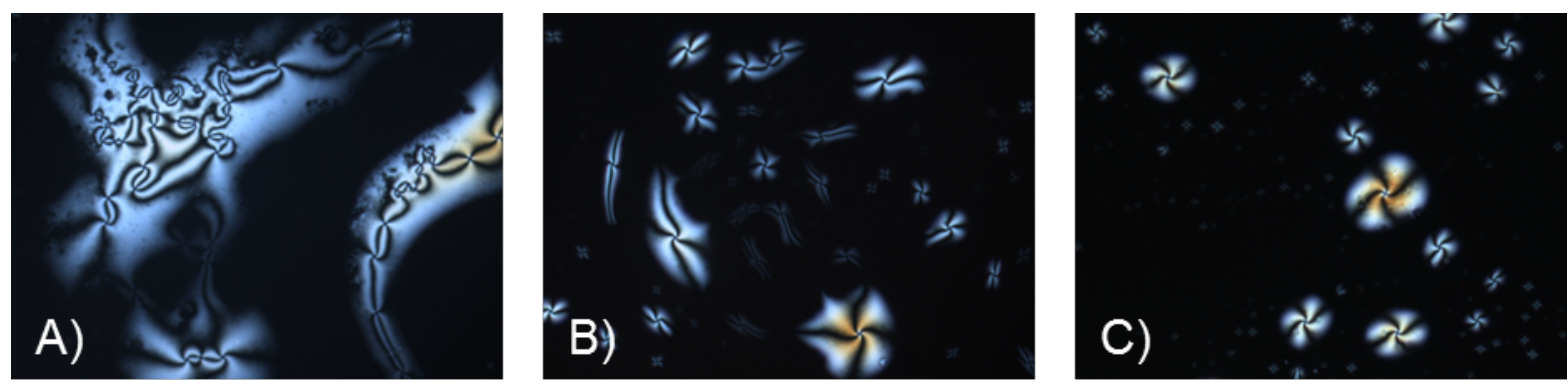

Figure 3: Schlieren textures of $11 \mathrm{a}$ and $11 \mathrm{~b}$ under crossed polarizers, upon cooling (cooling rate $5 \mathrm{~K} / \mathrm{min}$ ) from the isotropic liquid (magnification $200 \times)$ : (A) $11 \mathrm{a}\left(20^{\circ} \mathrm{C}\right),(B) 11 \mathrm{a}\left(14^{\circ} \mathrm{C}\right),(\mathrm{C}) 11 \mathrm{~b}\left(29^{\circ} \mathrm{C}\right)$. 

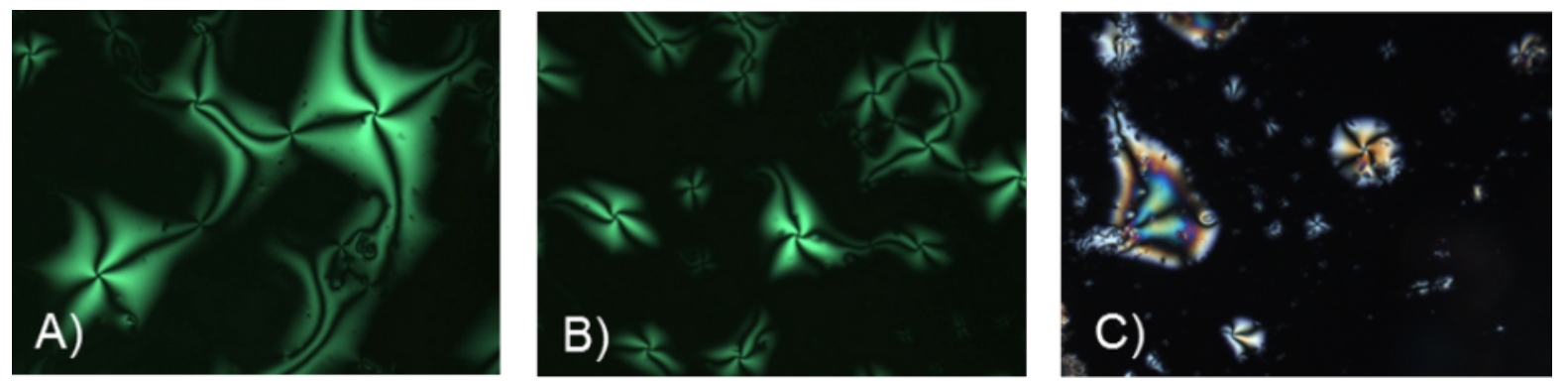

Figure 4: Schlieren textures of $13 \mathrm{a}$ and $\mathbf{1 3 b}$ under crossed polarizers upon cooling (cooling rate $5 \mathrm{~K} / \mathrm{min}$ ) from the isotropic liquid (magnification 200x): (A) $13 \mathrm{~b}\left(61^{\circ} \mathrm{C}\right),(B) 13 b$ at $61^{\circ} \mathrm{C}$, different section, (C) $13 \mathrm{a}\left(46^{\circ} \mathrm{C}\right)$.

Schlieren textures with fourfold brushes were observed for compound 13b at the transition from the isotropic liquid into the nematic phase. Quite similar textures were published by Dierking [40,41]. The areas without birefringence in Figure 3 and Figure 4 derive from homeotropic alignment of the molecules.

\section{X-ray diffraction studies}

The assignment of the nematic mesophases were exemplarily confirmed by wide-angle X-ray scattering (WAXS) experiments on compound 11a. Representative 2D diffractograms of the crystalline phase, the isotropic phase and the nematic phase are shown in Figure 5.

In the isotropic phase (Figure 5, part B) only a diffuse symmetric halo is observed. The diffraction pattern of 11a at $15{ }^{\circ} \mathrm{C}$ (Figure 5, part C) displays a halo split into two diffuse, crescent reflections, which is typical for nematic mesophases [42].

\section{Conclusion}

The mesogenic 4-cyano-1,1'-biphenyl group can be attached to either a malonate or a cyanoacetic ester scaffold by means of simple reaction sequences and with the aid of cheap chemical precursors. Linked ester molecules 11a,b and 13a,b have a distinct linear shape and easily form monotropic mesophases at ambient temperature upon cooling from the isotropic liquid. Following POM and X-ray studies, nematic mesophases could be assigned to all the described compounds 11a,b and 13a,b.

\section{Experimental \\ General information}

All reactions were carried out under a nitrogen atmosphere with Schlenk-type glassware. Solvents were dried and distilled under nitrogen prior to use. Flash chromatography was performed on silica gel, with grain size 40-63 $\mu \mathrm{m}$ (Macherey-Nagel).

The following instruments were used for physical characterization of the compounds. Elemental analyses: Carlo Erba Strumentazione Elemental Analyzer, Modell 1106. NMR: Bruker ARX-500 $\left({ }^{1} \mathrm{H}, 500 \mathrm{MHz} ;{ }^{13} \mathrm{C}, 125 \mathrm{MHz}\right)$. Assignments of the resonances are supported by $2 \mathrm{D}$ experiments and chemical shift calculations. ${ }^{1} \mathrm{H}$ and ${ }^{13} \mathrm{C}$ NMR spectra were referenced to an internal $\mathrm{Me}_{4} \mathrm{Si}$ (TMS) standard. IR: Bruker 22 FT-IR spectrometer with a golden-gate single-reflection diamond ATR system. MS: Bruker Daltonics mikro-TOF-Q (ESIMS). Differ-
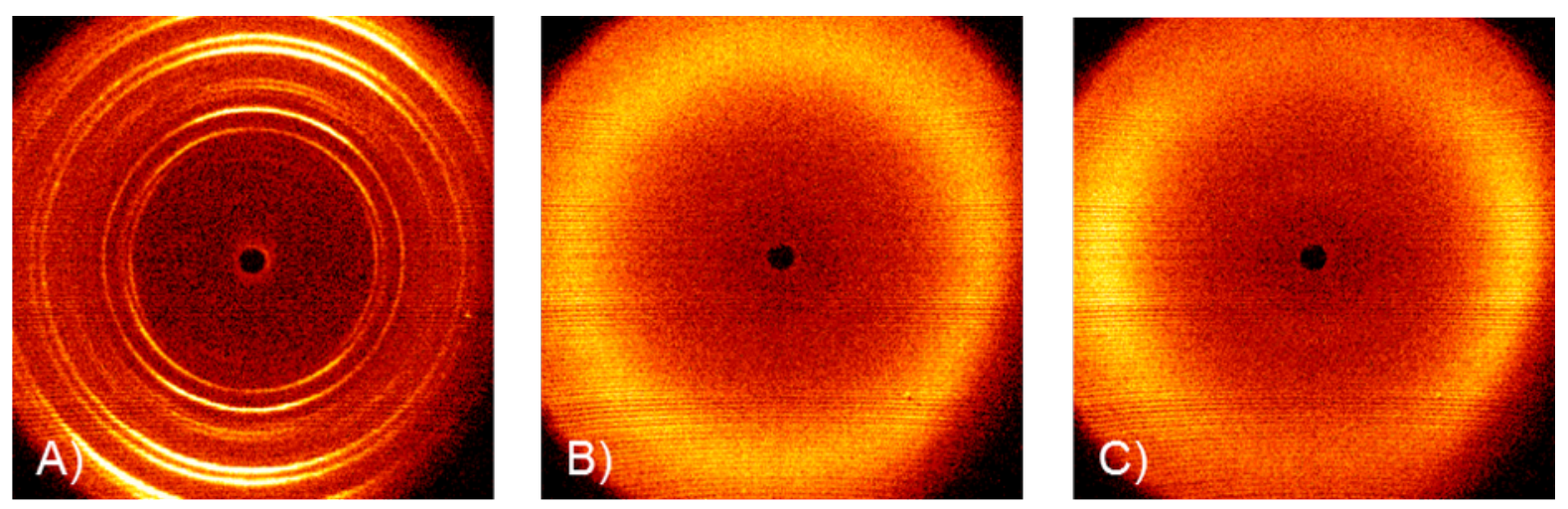

Figure 5: 2D X-ray scattering patterns of $11 \mathrm{a}$ : (A) crystalline phase at $50^{\circ} \mathrm{C},(\mathrm{B})$ isotropic phase at $25^{\circ} \mathrm{C}$, and (C) nematic phase at $15{ }^{\circ} \mathrm{C}$. 
ential scanning calorimetry (DSC): Mettler-Toledo DSC 822e (heating/cooling rates were 5 or $10 \mathrm{~K} \cdot \mathrm{min}^{-1}$ ). Polarizing optical microscopy: Olympus BX50 polarizing microscope combined with a Linkam TP93 central controller. X-ray diffraction (WAXS): Bruker AXS Nanostar C diffractometer employing Ni-filtered $\mathrm{Cu} \mathrm{K} \alpha$ radiation $(\lambda=1.5418 \AA$ ).

\section{6-[(4'-cyano-[1,1'-biphenyl]-4-yl)oxy]hexyl methyl malonate (11a)}

Pyridine (150 mg, $1.69 \mathrm{mmol})$ and then methyl 3-chloro-3oxopropionate (10) $(114 \mathrm{mg}, 0.84 \mathrm{mmol})$ were added over 10 min at $0{ }^{\circ} \mathrm{C}$ under a $\mathrm{N}_{2}$ atmosphere to a solution of 4 '-((6hydroxyhexyl)oxy)-[1,1'-biphenyl]-4-carbonitrile (9a) (500 mg, $1.69 \mathrm{mmol})$ in abs. $\mathrm{CH}_{2} \mathrm{Cl}_{2}(5 \mathrm{~mL})$. The reaction mixture was stirred at $0{ }^{\circ} \mathrm{C}$ for $1 \mathrm{~h}$, then for $2 \mathrm{~h}$ at rt. The reaction was quenched with $1 \mathrm{~N} \mathrm{H}_{2} \mathrm{SO}_{4}(3 \mathrm{~mL})$. The aqueous layer was extracted with $\mathrm{CH}_{2} \mathrm{Cl}_{2}(3 \times 5 \mathrm{~mL})$. The combined organic layers were washed with brine $(40 \mathrm{~mL})$, dried over $\mathrm{MgSO}_{4}$ and evaporated under reduced pressure. The crude product was purified by column chromatography on silica gel (hexanes/EtOAc 20:1) to give 11a as a colourless solid (188 $\mathrm{mg}, 0.48 \mathrm{mmol}$, 57\%). Mp $49.1{ }^{\circ} \mathrm{C} ;{ }^{1} \mathrm{H}$ NMR (500 MHz, $\left.\mathrm{CDCl}_{3}\right) \delta 1.41-1.47$ (m, 2H, 6-H), 1.49-1.55 (m, 2H, 7-H), 1.67-1.73 (m, 2H, 5-H), 1.79-1.85 (m, 2H, 8-H), 3.39 (s, 2H, 2-H), 3.74 (s, 3H, OCH $H_{3}$, 4.01 (t, $J=6.4 \mathrm{~Hz}, 2 \mathrm{H}, 9-\mathrm{H}), 4.17$ (t, $J=6.4 \mathrm{~Hz}, 2 \mathrm{H}, 4-\mathrm{H}), 6.98$ (d, $\left.J=8.5 \mathrm{~Hz}, 2 \mathrm{H}, 2^{\prime}-\mathrm{H}\right), 7.52$ (d, $\left.J=8.5 \mathrm{~Hz}, 2 \mathrm{H}, 3^{\prime}-\mathrm{H}\right), 7.64$ (d, $\left.J=8.5 \mathrm{~Hz}, 2 \mathrm{H}, 6^{\prime}-\mathrm{H}\right), 7.69$ (d, $\left.J=8.5 \mathrm{~Hz}, 2 \mathrm{H}, 7^{\prime}-\mathrm{H}\right) \mathrm{ppm}$; ${ }^{13} \mathrm{C}$ NMR $\left(125 \mathrm{MHz}, \mathrm{CDCl}_{3}\right) \delta 25.6,25.7$ (C-6, C-7), 28.4 (C-5), $29.0(\mathrm{C}-8), 41.4(\mathrm{C}-2), 52.5\left(\mathrm{OCH}_{3}\right), 65.6(\mathrm{C}-4), 67.8$ (C-9), $110.0\left(\mathrm{C}-8^{\prime}\right), 115.0\left(\mathrm{C}-2^{\prime}\right), 119.1(\mathrm{CN}), 127.0\left(\mathrm{C}-6^{\prime}\right)$, 128.3 (C-3'), 131.3 (C-5'), 132.6 (C-7'), 145.3 (C-4'), 159.7 (C-1'), 166.6 (C-3), 167.0 (C-1) ppm; ATR-FTIR $\tilde{v}: 2936$ (m), 2858 (w), 2224 (m), 1967 (w), 1730 (s), 1602 (m), 1494 (m), $1246(\mathrm{~s}), 1014(\mathrm{~m}), 822(\mathrm{~s})$; $\operatorname{ESIMS}(\mathrm{m} / \mathrm{z}): 343.1[\mathrm{M}+\mathrm{K}]^{+}$, $418.1[\mathrm{M}+\mathrm{Na}]^{+}, 396.1[\mathrm{M}+\mathrm{H}]^{+}, 278.15\left[\mathrm{C}_{19} \mathrm{H}_{20} \mathrm{NO}\right]^{+}$; Anal. calcd for $\mathrm{C}_{23} \mathrm{H}_{25} \mathrm{NO}_{5}$ : C, 69.86; H, 6.37; N, 3.54; found: $\mathrm{C}$, 69.47; H, 6.37; N, 3.47; $R_{\mathrm{f}} 0.56$ (hexanes/EtOAc 2:1).

\section{0-[(4'-cyano-[1,1'-biphenyl]-4-yl)oxy]decyl methyl malonate (11b)}

The ester 11b was obtained by the same procedure as described above for 11a from 4'-((10-hydroxydecyl)oxy)-[1,1'-biphenyl]4-carbonitrile (9b) (560 mg, $1.60 \mathrm{mmol})$, methyl 3-chloro-3oxopropionate (10) $(109 \mathrm{mg}, 0.80 \mathrm{mmol})$ and pyridine $(126 \mathrm{mg}$, $1.60 \mathrm{mmol})$ in abs. $\mathrm{CH}_{2} \mathrm{Cl}_{2}(5 \mathrm{~mL})$. The crude product was purified by column chromatography on silica gel (gradient: hexanes/EtOAc, 20:1, then 15:1) to give $\mathbf{1 1 b}$ as a colourless solid (240 mg, $0.53 \mathrm{mmol}, 65 \%)$. Mp $63.4{ }^{\circ} \mathrm{C}$; ${ }^{1} \mathrm{H}$ NMR $\left(500 \mathrm{MHz}, \mathrm{CDCl}_{3}\right) \delta 1.31-1.35(\mathrm{~m}, 10 \mathrm{H}, 6-\mathrm{H}, 7-\mathrm{H}, 8-\mathrm{H}, 9-\mathrm{H}$, $10-\mathrm{H}), 1.44-1.50(\mathrm{~m}, 2 \mathrm{H}, 11-\mathrm{H}), 1.61-1.67(\mathrm{~m}, 2 \mathrm{H}, 5-\mathrm{H})$ $1.77-1.83(\mathrm{~m}, 2 \mathrm{H}, 12-\mathrm{H}), 3.38(\mathrm{~s}, 2 \mathrm{H}, 2-\mathrm{H}), 3.75(\mathrm{~s}, 3 \mathrm{H}$,
$\left.\mathrm{OCH}_{3}\right), 4.00(\mathrm{t}, J=6.5 \mathrm{~Hz}, 2 \mathrm{H}, 13-\mathrm{H}), 4.14(\mathrm{t}, J=6.8 \mathrm{~Hz}, 2 \mathrm{H}$, 4-H), 6.98-6.99 (m, 2H, 2'-H), 7.51-7.53 (m, 2H, 3'-H), 7.63-7.64 (m, 2H, 6'-H), 7.68-7.69 (m, 2H, 7'-H) ppm; ${ }^{13} \mathrm{C}$ NMR (125 MHz, $\mathrm{CDCl}_{3}$ ) $\delta 25.7$ (C-6), 26.0 (C-11), 28.4 (C-5), 29.15 (C-12), 29.21, 29.33, 29.41, 29.45 (C-7, C-8, C-9, $\mathrm{C}-10), 41.4(\mathrm{C}-2), 52.4\left(\mathrm{OCH}_{3}\right), 65.7$ (C-4), 68.1 (C-13), 110.0 $\left(\mathrm{C}-8^{\prime}\right), 115.0\left(\mathrm{C}-2^{\prime}\right), 119.1(\mathrm{CN}), 127.0\left(\mathrm{C}-6^{\prime}\right), 128.3\left(\mathrm{C}-3^{\prime}\right)$, 131.3 (C-5'), 132.6 (C-7'), 145.3 (C-4'), 159.8 (C-1'), 166.6 (C-3), 167.0 (C-1) ppm; ATR-FTIR ṽ: $2927(\mathrm{~m}), 2854(\mathrm{w})$, 2225 (m), 1735 (s), 1603 (m), 1494 (m), 1249 (s), 1180 (m), $903(\mathrm{~m}), 823(\mathrm{~m})$; $\operatorname{ESIMS}(\mathrm{m} / \mathrm{z}): 474.2[\mathrm{M}+\mathrm{Na}]^{+}, 452.2[\mathrm{M}+$ $\mathrm{H}]^{+}$; Anal. calcd for $\mathrm{C}_{27} \mathrm{H}_{33} \mathrm{NO}_{5}$ : C, 71.82; H, 7.37; N, 3.10; found: C, 71.66; H, 7.34; N, 3.03; $R_{\mathrm{f}} 0.76$ (hexanes/EtOAc 2:1).

\section{6-[(4'-cyano-[1,1'-biphenyl]-4-yl)oxy]hexyl 2-cyanoacetate (13a)}

To a solution of 4'-((6-hydroxyhexyl)oxy)-[1,1'-biphenyl]-4carbonitrile (9a) $(100 \mathrm{mg}, 338 \mu \mathrm{mol})$ in abs. $\mathrm{CH}_{2} \mathrm{Cl}_{2}(2.5 \mathrm{~mL})$ were added sequentially a solution of cyanoacetic acid (12) (32 mg, $376 \mu \mathrm{mol})$ in EtOAc $(0.4 \mathrm{~mL})$, a solution of DMAP $(12 \mathrm{mg}, 98 \mu \mathrm{mol})$ in abs. $\mathrm{CH}_{2} \mathrm{Cl}_{2}(0.8 \mathrm{~mL})$ and then at $0{ }^{\circ} \mathrm{C} \mathrm{a}$ solution of dicyclohexylcarbodiimide $(77 \mathrm{mg}, 376 \mu \mathrm{mol})$ in abs. $\mathrm{CH}_{2} \mathrm{Cl}_{2}(2.5 \mathrm{~mL})$. The reaction mixture was stirred at $\mathrm{rt}$ for $7.5 \mathrm{~h}$, then evaporated under vacuum. The crude product was purified by column chromatography on silica gel (hexanes/ EtOAc 12:1) to give 13a as a colourless solid (72 mg, $190 \mu \mathrm{mol}, 59 \%) . \mathrm{Mp} 89.0^{\circ} \mathrm{C} ;{ }^{1} \mathrm{H}$ NMR $\left(500 \mathrm{MHz}, \mathrm{CDCl}_{3}\right) \delta$ $1.43-1.49$ (m, 2H, 6-H), 1.51-1.56 (m, 2H, 7-H), 1.71-1.76 (m, 2H, 5-H), 1.80-1.85 (m, 2H, 8-H), 3.45 (s, 2H, 2-H), 4.01 (t, $J$ $=6.4 \mathrm{~Hz}, 2 \mathrm{H}, 9-\mathrm{H}), 4.23(\mathrm{t}, J=6.3 \mathrm{~Hz}, 2 \mathrm{H}, 4-\mathrm{H}), 6.98-6.99(\mathrm{~m}$, $\left.2 \mathrm{H}, 2^{\prime}-\mathrm{H}\right), 7.52-7.53$ (m, 2H, 3'-H), 7.63-7.64 (m, 2H, 6'-H), 7.68-7.69 (m, 2H, 7'-H) ppm; ${ }^{13} \mathrm{C}$ NMR (125 MHz, $\left.\mathrm{CDCl}_{3}\right) \delta$ 24.7 (C-2), 25.5 (C-6), 25.6 (C-7), 28.3 (C-5), 29.0 (C-8), 66.9 (C-4), 67.8 (C-9), 110.0 (C-8'), 112.9 (C-1), 115.0 (C-2'), 119.1 $(C \mathrm{~N}), 127.0$ (C-6'), 128.3 (C-3'), 131.3 (C-5'), 132.5 (C-7'), 145.2 (C-4'), 159.6 (C-1'), 162.9 (C-3) ppm; ATR-FTIR $\tilde{v}$ : 2941 (m), 2866 (w), 2225 (w), 1746 (m), 1602 (m), 1494 (m), 1249 (m), $1180(\mathrm{~m}), 903$ (s), 723 (s); ESIMS (m/z): $385.1[\mathrm{M}+$ $\mathrm{Na}]^{+}, 363.1[\mathrm{M}+\mathrm{H}]^{+}$; Anal. calcd for $\mathrm{C}_{22} \mathrm{H}_{22} \mathrm{~N}_{2} \mathrm{O}_{3}$ : C, 71.91; $\mathrm{H}, 6.12 ; \mathrm{N}, 7.57$; found: C, 71.44; H, 6.06; N, 7.73; $R_{\mathrm{f}} 0.68$ (hexanes/EtOAc 2:1).

\section{0-[(4'-cyano-[1,1'-biphenyl]-4-yl)oxy]decyl 2-cyanoacetate (13b)}

The cyanoacetic ester 13b was obtained by the same procedure as described above for 13a from 4'-((10-hydroxydecyl)oxy)[1,1'-biphenyl]-4-carbonitrile (9b) (120 mg, $341 \mu \mathrm{mol})$, cyanoacetic acid (12) (32 mg, $376 \mu \mathrm{mol})$, DMAP (13 mg, $102 \mu \mathrm{mol})$, and dicyclohexylcarbodiimide $(77 \mathrm{mg}, 376 \mu \mathrm{mol})$ in abs. $\mathrm{CH}_{2} \mathrm{Cl}_{2}(6.5 \mathrm{~mL})$. The crude product was purified by column chromatography on silica gel (hexanes/EtOAc 10:1) to give $\mathbf{1 3 b}$ 
as a colourless solid (57 mg, $140 \mu \mathrm{mol}, 41 \%$ ). Mp $93.2{ }^{\circ} \mathrm{C}$; ${ }^{1} \mathrm{H}$ NMR $\left(500 \mathrm{MHz}, \mathrm{CDCl}_{3}\right) \delta 1.32-1.36(\mathrm{~m}, 10 \mathrm{H}, 6-\mathrm{H}, 7-\mathrm{H}$, 8-H, 9-H, 10-H), 1.44-1.50 (m, 2H, 11-H), 1.65-1.71 (m, 2H, $5-\mathrm{H}), 1.78-1.83(\mathrm{~m}, 2 \mathrm{H}, 12-\mathrm{H}), 3.44(\mathrm{~s}, 2 \mathrm{H}, 2-\mathrm{H}), 4.00(\mathrm{t}, J=$ $6.5 \mathrm{~Hz}, 2 \mathrm{H}, 13-\mathrm{H}), 4.20(\mathrm{t}, J=6.9 \mathrm{~Hz}, 2 \mathrm{H}, 4-\mathrm{H}), 6.98-6.99$ (m, $\left.2 \mathrm{H}, 2^{\prime}-\mathrm{H}\right), 7.51-7.53\left(\mathrm{~m}, 2 \mathrm{H}, 3^{\prime}-\mathrm{H}\right), 7.63-7.64\left(\mathrm{~m}, 2 \mathrm{H}, 6^{\prime}-\mathrm{H}\right)$, 7.68-7.69 (m, 2H, 7'-H) ppm; ${ }^{13} \mathrm{C}$ NMR (125 MHz, $\left.\mathrm{CDCl}_{3}\right) \delta$ 24.7 (C-2), 25.6 (C-6), 26.0 (C-11), 28.3 (C-5), 29.11 (C-12), 29.21, 29.32, 29.36, 29.40 (C-7, C-8, C-9, C-10), 67.1 (C-4), 68.1 (C-13), 110.0 (C-8'), 113.0 (C-1), 115.0 (C-2'), 119.1 (CN), 127.0 (C-6'), 128.3 (C-3'), 131.3 (C-5'), 132.5 (C-7'), 145.2 (C-4'), 159.7 (C-1'), 162.9 (C-3) ppm; ATR-FTIR v : 2928 (m), 2855 (w), 2225 (w), 1747 (m), 1603 (m), 1494 (m), $1250(\mathrm{~m}), 1180(\mathrm{~m}), 903(\mathrm{~s}), 725(\mathrm{~s}) ; \operatorname{ESIMS}(\mathrm{m} / \mathrm{z}): 457.1[\mathrm{M}+$ $\mathrm{K}]^{+}, 441.2[\mathrm{M}+\mathrm{Na}]^{+}, 436.2,419.2[\mathrm{M}+\mathrm{H}]^{+}$; Anal. calcd for $\mathrm{C}_{26} \mathrm{H}_{30} \mathrm{~N}_{2} \mathrm{O}_{3}$ : C, 74.61; H, 7.22; N, 6.69; found: C, 74.04; $\mathrm{H}$, $7.18 ; \mathrm{N}, 6.54 ; R_{\mathrm{f}} 0.58$ (hexanes/EtOAc 2:1).

\section{Supporting Information}

\section{Supporting Information File 1}

Full experimental procedures and DSC traces of $\mathbf{1 1 b}$ and $13 b$.

[http://www.beilstein-journals.org/bjoc/content/ supplementary/1860-5397-8-40-S1.pdf]

\section{Acknowledgements}

Generous financial support by the Bundesministerium für Bildung und Forschung, the Ministerium für Wissenschaft, Forschung und Kunst des Landes Baden-Württemberg and the Max-Planck-Gesellschaft (International Max Planck Research School for Advanced Materials fellowship for M.K.) is gratefully acknowledged. We would like to thank Ingo Dierking for helpful discussions and suggestions.

\section{References}

1. Goodby, J. W. In Handbook of Liquid Crystals; Demus, D.; Goodby, J.; Gray, G. W.; Spiess, H.-W.; Vill, V., Eds.; Wiley-VCH: Weinheim, Germany, 1998; Vol. 2A, pp 3-21.

2. Assanto, G.; Peccianti, M. Mol. Cryst. Liq. Cryst. 2008, 488, 163-178. doi:10.1080/15421400802240540

3. Eidenschink, R. Mol. Cryst. Liq. Cryst. 1983, 94, 119-125. doi:10.1080/00268948308084251

4. Raviol, A.; Stille, W.; Strobl, G. J. Chem. Phys. 1993, 105, 3788-3794. doi:10.1063/1.470057

5. O'Neill, M.; Kelly, S. M. Adv. Mater. 2003, 15, 1135-1146. doi:10.1002/adma.200300009

6. O'Neill, M.; Kelly, S. M. Adv. Mater. 2011, 23, 566-584. doi:10.1002/adma.201002884

7. Kopp, V. I.; Fan, B.; Vithana, H. K. M.; Genack, A. Z. Opt. Lett. 1998, 23, 1707-1709. doi:10.1364/OL.23.001707
8. Dąbrowski, R. Mol. Cryst. Liq. Cryst. 2004, 421, 1-21. doi:10.1080/15421400490501112

9. Kirsch, P.; Bremer, M. Angew. Chem. 2000, 112, 4384-4405. doi:10.1002/1521-3757(20001201)112:23<4384::AID-ANGE4384>3.0. CO;2-S

Angew. Chem., Int. Ed. 2000, 39, 4216-4235

doi:10.1002/1521-3773(20001201)39:23<4216::AID-ANIE4216>3.0.CO ;2-K

10. Bisoyi, H. K.; Kumar, S. Chem. Soc. Rev. 2010, 39, 264-285. doi:10.1039/b901792p

11. Goodby, J. W. Liq. Cryst. 2011, 38, 1363-1387. doi:10.1080/02678292.2011.614700

12. Shimizu, Y.; Oikawa, K.; Nakayama, K.-i.; Guillon, D. J. Mater. Chem. 2007, 17, 4223-4229. doi:10.1039/b705534j

13. Yang, S. H.; Hsu, C.-S. J. Polym. Sci., Part A: Polym. Chem. 2009, 47, 2713-2733. doi:10.1002/pola.23342

14. Gray, G. W.; Kelly, S. M. J. Mater. Chem. 1999, 9, 2037-2050. doi:10.1039/a902682g

15. Bulkin, B. J.; Rose, R. K.; Santoro, A. Mol. Cryst. Liq. Cryst. 1977, 43, 53-58. doi:10.1080/00268947708084934

16. Giroud-Godquin, A. M. In Handbook of Liquid Crystals; Demus, D.; Goodby, J.; Gray, G. W.; Spiess, H.-W.; Vill, V., Eds.; Wiley-VCH: Weinheim, Germany, 1998; Vol. 2B, pp 901-932.

17. Mühlberger, B.; Haase, W. Liq. Cryst. 1989, 5, 251-263. doi:10.1080/02678298908026368

18. Dzhabarov, V. I.; Knyazev, A. A.; Strelkov, M. V.; Molostova, E. Y.; Schustov, V. A.; Haase, W.; Galyametdinov, Y. G. Liq. Cryst. 2010, 37, 285-291. doi:10.1080/02678290903506040

See for recent examples of liquid crystalline $\beta$-diketonate metal complexes.

19. Santoro, A.; Whitwood, A. C.; Williams, J. A. G.; Kozhevnikov, V. N.; Bruce, D. W. Chem. Mater. 2009, 21, 3871-3882. doi:10.1021/cm9012156

See for recent examples of liquid crystalline $\beta$-diketonate metal complexes.

20. Pucci, D.; Aiello, I.; Aprea, A.; Bellusci, A.; Crispini, A.; Ghedini, M. Chem. Commun. 2009, 1550-1552. doi:10.1039/b818603k See for recent examples of liquid crystalline $\beta$-diketonate metal complexes.

21. Yoshida, J.; Sato, H.; Hoshino, N.; Yamagishi, Y. J. Phys. Chem. B 2008, 112, 9677-9683. doi:10.1021/jp8011206 See for recent examples of liquid crystalline $\beta$-diketonate metal complexes.

22. Knyazev, A. A.; Dzhavarov, V. I.; Lapaev, D. V.; Lopkov, V. S.; Haase, W.; Galyametdinov, Y. G. Russ. J. Gen. Chem. 2010, 80, 756-760. doi:10.1134/S1070363210040122

23. Han, J.; Guo, H.; Wang, X.-G.; Pang, M.-L.; Meng, J.-B. Chin. J. Chem. 2007, 25, 129-131. doi:10.1002/cjoc.200790007

24. Han, J.; Zhang, L. F.; Wan, W. Chin. Chem. Lett. 2004, 15, 155-158.

25. Farago, M. E.; Amirhaeri, S. Inorg. Chim. Acta 1984, 81, 205-212. doi:10.1016/S0020-1693(00)88759-9

26. Lose, D.; Diele, S.; Pelzl, G.; Dietzmann, E.; Weissflog, W. Liq. Cryst. 1998, 24, 707-717. doi:10.1080/026782998206821

27. Maringa, N.; Lenoble, J.; Donnio, B.; Guillon, D.; Deschenaux, R. J. Mater. Chem. 2008, 18, 1524-1534. doi:10.1039/b717105f

28. Dardel, B.; Guillon, D.; Heinrich, B.; Deschenaux, R. J. Mater. Chem. 2001, 11, 2814-2831. doi:10.1039/b103798f

29. Chuard, T.; Dardel, B.; Deschenaux, R.; Even, M. Carbon 2000, 38 , 1573-1576. doi:10.1016/S0008-6223(99)00286-9 
30. Chuard, T.; Deschenaux, R.; Hirsch, A.; Schönberger, H. Chem. Commun. 1999, 2103-2104. doi:10.1039/a905058b

31. Deschenaux, R.; Even, M.; Guillon, D. Chem. Commun. 1998, 537-538. doi:10.1039/a709092g

32. Schubert, H.; Hoffmann, S.; Hauschild, J.; Marx, I. Z. Chem. 1977, 17, 414-415. doi:10.1002/zfch.19770171106

33. Kašpar, M.; Sverenyák, H.; Hamplová, V.; Pakhomov, S. A.; Glogarová, M. Mol. Cryst. Liq. Cryst. Sci. Technol., Sect. A 1995, 260, 241-246. doi:10.1080/10587259508038695

34. Pintre, I. C.; Serrano, J. L.; Blanca Ros, M.; Martínez-Perdiguero, J.; Alonso, I.; Ortega, J.; Folcia, C. L.; Etxebarria, J.; Alicante, R.; Villacampa, B. J. Mater. Chem. 2010, 20, 2965-2971. doi:10.1039/b923616c

35. Chong, J. M.; Heuft, M. A.; Rabbat, F. J. Org. Chem. 2000, 65, 5837-5838. doi:10.1021/jo000291u

36. Sahade, D. A. Polymerizable Liquid Crystal Compound, Polymerizable Liquid Crystal Composition, and Alignment Film. EP 2062882, May 27, 2009.

Chem. Abstr. 2008, 149, 91567.

37. Zugenmaier, P. Liq. Cryst. 2002, 29, 443-448. doi:10.1080/02678290110113522

38. Nakatsuji, S.; Ikemoto, H.; Akutsu, H.; Yamada, J.-i.; Mori, A. J. Org. Chem. 2003, 68, 1708-1714. doi:10.1021/jo0206972

39. Kadkin, O. N.; Han, H.; Galyametdinov, Y. G. J. Organomet. Chem. 2007, 692, 5571-5582. doi:10.1016/j.jorganchem.2007.09.010

40. Dierking, I. Textures of Liquid Crystals; Wiley-VCH: Weinheim, Germany, 2003. doi:10.1002/3527602054

41. Dierking, I. Physik Journal 2009, 8 (4), 27-32.

42. Kuiper, S.; Norder, B.; Jager, W. F.; Dingemans, T. J.; van Turnhout, J.; Picken, S. J. J. Phys. Chem. B 2011, 115, 1416-1421. doi:10.1021/jp105643s

\section{License and Terms}

This is an Open Access article under the terms of the Creative Commons Attribution License (http://creativecommons.org/licenses/by/2.0), which permits unrestricted use, distribution, and reproduction in any medium, provided the original work is properly cited.

The license is subject to the Beilstein Journal of Organic Chemistry terms and conditions:

(http://www.beilstein-journals.org/bjoc)

The definitive version of this article is the electronic one which can be found at: $\underline{\text { doi:10.3762/bjoc. } 8.40}$ 